\title{
Effective Attacks In The Salvo Combat Model: Salvo Sizes And Quantities Of Targets ${ }^{1}$
}

\author{
Michael J Armstrong \\ Sprott School of Business, Carleton University \\ Ottawa, ON, K1S 5B6, Canada
}

\begin{abstract}
This article considers two related questions of tactics in the context of the salvo model for naval missile combat. For a given set of targets, how many missiles should be fired to produce an effective attack? For a given available salvo size, how many enemy targets should be fired at? In the deterministic version of the model I derive a simple optimality relationship between the number of missiles to fire and the number of targets to engage. In the stochastic model I employ the expected loss inflicted and the probability of enemy elimination as the main performance measures, and use these to derive salvo sizes that are in some sense "optimal". I find that the offensive firepower needed for an effective attack depends not only on a target's total strength but also on the relative balance between its active defensive power and passive staying power.
\end{abstract}

Keywords: military modeling, naval tactics, salvo combat, applied probability.

\section{Introduction}

When preparing for a battle involving modern warships, "...the first question to answer regarding tactical configuration is how much striking power would be sufficient to eliminate the enemy at one blow" [5: p 291]. A preliminary response to this question became possible with the development of the deterministic salvo combat model by Hughes in [6] in which the exchange of fire consists of discrete salvos of missiles. This aggregated model of naval missile combat captures in a simple way the interaction between offensive firepower (e.g., Harpoon anti-shipping cruise missiles: ASCMs) and defensive firepower (e.g., Standard surface-to-air missiles: SAMs). The nature of this interaction depends on the relative balance of offensive and defensive weaponry used by the ships on each side, i.e., the combatants' lethality, as defined in [2].

One limitation of the deterministic salvo model is that it makes no provision for variation or uncertainty in combat results. Consequently, Armstrong in [1] developed a stochastic version of the model that includes expressions for the mean and variance of losses by each force. This extension improves the realism of the salvo model, but it also shows that some tactical issues are not as straightforward as the deterministic model implies. For example, the deterministic model suggests that a force of "adequate" size is sufficient to guarantee the effectiveness of an attack. By contrast, the stochastic model

\footnotetext{
1 This is the peer reviewed version of the following article: Armstrong MJ, 2007, "Effective attacks in the salvo combat model: salvo sizes and quantities of targets", Naval Research Logistics 54 \#1, 66-77, DOI: 10.1002/nav.20187, which has been published in final form at http://onlinelibrary.wiley.com/doi/10.1002/nav.20187/abstract. This article may be used for noncommercial purposes in accordance with Wiley Terms and Conditions for Self-Archiving.
} 
illustrates (see, e.g., [3]) that victory by a merely "adequate" force is far from assured, though its probability can be improved by committing more forces.

This brings me back to the opening quote about the use of "sufficient" force for an attack and thus to the pair of research questions that I deal with herein. Against a given enemy, how many missiles should I fire in order to ensure an "effective" attack? Conversely, given a limited salvo size, how many enemy targets should I engage?

The open literature contains a small amount of work related to this topic. One such study [8] incorporates the deterministic salvo equations into a larger simulation model in which each side has several identical ships operating independently. Although all of the ships are within offensive missile range of each other, they are far enough apart to target separately, and each one defends individually. The simulation assumes that ASCMs are aimed at randomly chosen enemy ships, but that all other features of combat are deterministic. The study notes that although generally it is beneficial to allocate fire across all opposing ships, there are certain cases in which it is better to concentrate fire against just a portion of the enemy fleet.

Another related work [7] is a brief numerical study of attacks against a "high value unit" such as an aircraft carrier that is being escorted by other vessels such as frigates. That work assumes that all features of combat are deterministic, except that each non-intercepted ASCM will acquire and hit a randomly selected ship, i.e., either the high value unit or an escort. The study's goal is to estimate the salvo size needed to provide an $80 \%$ chance of eliminating the high value unit.

Also of some relevance is the body of work on firing disciplines such as "shootlook-shoot" (see e.g., [9: Ch 13]). The focus in these studies tends to be more on the tradeoff between inflicting damage ("shooting") and assessing damage ("looking") for individual weapons over the course of an extended battle, rather than on questions about a coordinated salvo attack by a group of units.

In this article I examine the issue of attack effectiveness in the context of both the deterministic and the stochastic versions of the salvo combat model. I take the viewpoint of an attack planner who must decide how many missiles to fire and/or how many targets to shoot at. The intended attack could be one-half of a full battle, in which friendly ships attack the enemy while they simultaneously counter-attack. Alternatively it could be a "one-way" strike, where my side only attacks and the enemy only defends, for example in a surprise air strike with stand-off missiles against an enemy surface force.

The next section reviews the structure of the salvo combat model and then begins analysis of the deterministic model. For this relatively simple case I derive an exact optimality relationship between salvo sizes and target quantities for effective attacks.

The remainder of the study considers attack effectiveness in the more complex stochastic model. I use a combination of analytical and numerical methods to evaluate two principle measures of effectiveness: the expected loss inflicted on the enemy, and the 
probability of elimination of the target. Here I draw parallels with stochastic inventory models (see, e.g., [4: Ch 11]), where the approximately equivalent metrics are the expected fill rate (the average proportion of demands that are satisfied during a period) and the cycle service level (the probability that all demands are met during a period).

I use expected loss as the primary criterion for determining the number of targets to engage. The results suggest that the linear decision rule derived in the deterministic model provides a good approximation when used in the stochastic model against targets that are small in number or have "balanced" defenses: that is, targets that make equal use of active defensive power and passive staying power. When the targets are numerous and employ unbalanced defenses, the attacker can achieve better results by retaining the linear form of the heuristic but recalculating its parameters. This part of the study may be of particular interest for smaller navies facing more powerful opponents, as they may need to focus on a subset of potential targets in order to inflict any losses at all.

I subsequently use the probability of target elimination as a criterion for choosing the number of missiles to fire at each target. I show how to compensate for variability in combat by calculating a safety margin of extra missiles to fire (similar to safety stock in inventory theory) so as to provide any specified probability of success. This section may be of special interest for larger navies that expect to fight from a position of superiority.

One interesting outcome of this research is the derivation of an "optimal" salvo guideline for the stochastic model that depends on the kind of defenses employed. Against an enemy that relies mostly on active defensive power (due to, e.g., SAM systems) it is best to focus an attack on a smaller number of targets than what the deterministic model would suggest, and thereby assign each target a larger safety margin of extra missiles. Conversely, if the enemy ships rely more on passive staying power (due to, e.g., hull compartmentalization) then it is better to spread the attack across a larger number of targets, each of which may be allotted a negative safety margin of missiles. This difference in tactics holds even if the different ship types have the same total strength (i.e., passive plus active combined).

The paper concludes with a summary of the results and limitations of this study. The most important limitation of course is that all of the work herein is based upon the salvo models of [1] and [6]. The primary purpose of these models is to capture only the essential aspects of missile combat, thereby allowing researchers, students, and analysts to improve their understanding of how such battles are likely to behave. Thus my results are meaningful to real naval tacticians only to the extent that these simple models reflect real naval combat. An appendix contains the derivations of the paper's analytical results.

\section{The Salvo Model}

This section briefly describes the structure of the salvo combat model as used herein; the original publications [1,6] contain more detailed descriptions of the model's components and assumptions. Consider an impending battle between two naval forces, Red (Side A) and Blue (Side B). Red begins the battle by firing a simultaneous salvo of 
$N$ identical well-targeted offensive missiles (ASCMs) at the Blue force. Any missiles that are not well-targeted (e.g., that misfire or are poorly aimed) are ignored by this model. Blue has $B$ identical ships and each one has defensive power $z$, which is the number of incoming well-targeted enemy missiles intercepted per salvo by active defenses such as SAMs. Each Red missile that Blue does not intercept causes losses equal to a fraction of a ship $v$. The staying power $x=1 / v$ is the number of ASCM hits needed to achieve a firepower kill on one Blue ship; i.e., to put it out of action, though not necessarily sink it. The number of ships lost due to this attack is

$$
\operatorname{Loss}_{B}=\sum^{N e t A B} v, \quad 0 \leq \operatorname{Loss}_{B} \leq B, \quad \text { where } \quad \operatorname{Net}_{A B}=N-\sum^{B} z
$$

Here $N e t_{A B}$ represents the net number of good offensive missiles from Red that are not intercepted by Blue's defenses; [6] refers to this as "combat power".

In the original deterministic model from [6] all of the model parameters are constants. By contrast, the stochastic version of [1] allows each ship's defensive power $z$ to be an independent random variable; likewise, each offensive missile has an independent probability $p_{\alpha}$ of being well-targeted or "good", and the loss $v$ caused by each one is also an independent random variable. Under the stochastic model's assumptions, the resulting losses are approximated by a truncated normal distribution, and this is used to derive the mean, variance, and the probability of elimination. Note that estimating appropriate values for each of these parameters for real ships is not a trivial task; [3] provides one example of how it can done for an historical battle.

\section{The Deterministic Model}

This section examines the effect of salvo size within the deterministic model, first for one target and then for multiple targets.

\section{One Target}

Suppose that Red is planning an attack against a single Blue target and needs to determine the total number of missiles $N$ to launch towards it from one or more of their own ships or aircraft. This "target" could be an individual ship, or it could be several ships in close proximity that are fired upon as a group, as in [7]. For discussion purposes, let the target be a single Blue ship (so $B=1$ ) that can consistently intercept $z=3$ incoming ASCMs per salvo. The deterministic model only considers well-targeted or "good" ASCMs, so that any missile fired will hit the target if not intercepted. Also let each non-intercepted ASCM consistently cause the loss of $v=1 / 3$ ships, so that the targeted ship has a staying power of $x=3 / 1=3$ hits.

Assuming that Red's goal is to put enemy ships out of action (as opposed to, e.g., distracting Blue while performing some other task), it is clearly pointless to fire at this target with a salvo of size $N \leq z=3$ missiles, as they would all be intercepted by the defenses without causing any damage. Ideally Red should fire $N^{* *} \equiv(z+1 / v)=6$ 
ASCMs if possible so that the target is completely eliminated; any missiles beyond this "optimal" $N^{* *}$ value would be wasteful overkill. The solid line in Figure 1 illustrates this by plotting Blue's losses as a function of Red's salvo size $N$. The plot is piecewise linear in 3 sections: from 0 to 3 missiles the entire attack is intercepted, from 3 to 6 an increasing amount of damage is inflicted, while past 6 missiles there is no additional damage. The upward-sloping straight line between 3 and 6 illustrates that the marginal benefit from each added missile is constant within this range. For later use define the mid-point of this range as $N^{*} \equiv(z+1 /(2 v))=(z+x / 2)$, i.e., the salvo size needed to eliminate half of the target, in this case $N^{*}=4.5$ missiles.

\section{Multiple Targets}

Now suppose that there are several identical potential Blue targets that Red could attack, all spread far enough apart that they defend independently. As before, I will assume that Red's objective is to knock-out as many enemy ships as possible for any given total salvo size $N$. Notice that with defenses protecting each target, there is no point in shooting at a second target until enough missiles have been allocated to eliminate the first one. For example, if only $N=6 \mathrm{ASCM}$ are available, it is clearly better to fire all 6 at one target and thereby eliminate it than to fire 3 at each of two targets and have them all intercepted to no effect. Given a choice, an attacker is better off using a small incremental increase in salvo size to inflict more damage on the first target rather than wasting those missiles to pointless interception by a second target.

This is illustrated in Figure 1, where the dashed line shows the loss resulting from evenly dividing a salvo of total size $N$ between 2 targets. It is initially lower than the loss caused by concentrating fire on 1 target (the solid line) because there is twice as much defensive power to saturate, but it crosses over at $N=2 N^{*}=9$ and thereafter is higher. In this example, firing 9 missiles at 1 target will knock it out completely but leave the second target untouched; on the other hand, firing 5 missiles at one target and 4 at the other (an average of 4.5 each) will destroy $2 / 3$ of one and $1 / 3$ of the other and so yield the same total effect. For salvo sizes below this "breakpoint" number, Red does better by focusing on just 1 target, while for larger salvos they can inflict more damage by spreading their fire evenly across 2 targets. If Red has sufficient firepower, they ideally should fire a total salvo of $N=2 N^{* *}=12$ missiles ( 6 apiece) in order to eliminate both targets completely.

This breakpoint approach to determining how many targets to engage is generalized in the following proposition.

Proposition 1. The following relationships hold in the deterministic model when targets are identical:

(a) For a given number of targets $t=T$, a total salvo size of $N=T N^{* *}$ is optimal in the sense that it is the minimum salvo size that will completely eliminate all T targets; 
(b) For a given number of targets $t=T$, any total salvo size $N$ in the range $N_{T-1, T} \leq N \leq$ $N_{T, T+1}$ will inflict losses at least as large against $T$ targets as against either $T-1$ or $T+1$ targets, where $N_{t, t+1} \equiv\{z+(t)(z+1 / v)\}$ and so $N_{T-1, T}=N_{T, T+1}-N^{* *}$;

(c) For a given total salvo size $N$, the loss-maximizing number of targets to fire at is the minimum integer value of $t$ such that $t \geq(N-z) /(z+1 / v)$;

(d) Under these firing rules, the average number of missiles per target will always be at least $N^{*}$, and it will converge to $N^{* *}$ for large $N$ and $t$. That is,

$$
N / t \geq N^{*}=(z+1 /(2 v)), \text { and } N / t \rightarrow N^{* *}=(z+1 / v) \text { as } N \rightarrow \infty \text { and } t \rightarrow \infty \text {. }
$$

Likewise, the proportion of losses amongst t targeted units will at least equal (t-1)/t and will converge to $100 \%$ as $N \rightarrow \infty$ and $t \rightarrow \infty$.

Applying these analytical results to the earlier numerical example gives breakpoints of $N_{t, t+1}=\{9,15,21,27,33, \ldots\}$ that are separated by increments of $N^{* *}=6$ missiles. Thus a salvo of $N_{3,4}=21$ missiles inflicts the same losses whether it is divided between 3 targets or 4 . If I specifically choose to fire at say $t=3$ targets, then a total salvo size of between 15 and 21 missiles is appropriate; if there are less than 15 available to fire at once, I could inflict greater losses by focusing on fewer targets, whereas a salvo of more than 21 would be better fired against a larger number. If I am limited to a salvo of say size 11, then I should shoot at 2 targets, as $(11-3) /(3+3)=1.33$ which rounds up to 2. On a per-target basis, it is best to allocate 9 or less missiles against 1 target, from 4.5 to 7.5 at each of 2 targets, 5 to 7 at each of 3 targets, and so on, converging towards $N^{* *}$ $=6$ per target as the number of targets $t$ grows larger.

\section{The Stochastic Model: Expected Losses}

This section turns to the stochastic version of the salvo combat model, for which one sensible measure of attack effectiveness is the expected loss: i.e., the average number of enemy targets destroyed. This is similar to the analysis of inventory policies using their expected fill rate: i.e., the average proportion of customer demands satisfied.

\section{One Target}

When there is only a single enemy target it is possible to determine the behavior of the expected loss EL analytically.

Proposition 2. For a single target, the expected loss EL behaves as follows:

(a) EL is increasing in mean offensive firepower according to an s-shaped curve whose inflection point corresponds to EL=50\%, i.e., half elimination of the target;

(b) EL is increasing in the variation of offensive firepower when $E L<50 \%$, and is decreasing when EL> 50\%; 
(c) EL is increasing in salvo size $N$ according to an s-shaped curve whose inflection point lies at $N=N * / p_{\alpha}$ and corresponds to $E L=50 \%$.

For illustration, consider a stochastic version of the previous numerical example. Let the damage from each hit be random, with the same average $\mu_{v} \equiv \mathrm{E}[v]=1 / 3$ as before, but now with a standard deviation of 1/6 ships lost per hit. Let the defensive power also have the same average value of $E[z]=3$ as before but now with a standard deviation of 1.225. Likewise assume that each offensive missile has an independent probability $p_{\alpha}=$ 0.50 of success. In Figure 2, the solid curve shows the behavior of expected loss $E L$ with respect to salvo size $N$. The curve is convex increasing up to the inflection point at $N^{*} / p_{\alpha}$ $=(4.5) /(0.5)=9$ missiles, which is sufficient to give $E L=0.5$ targets. This inflection point also indicates the point of maximal marginal return per missile fired; thereafter the curve is concave increasing, indicating diminishing returns to further increases in salvo size. The curve is approximately inversely symmetric about the inflection point, in the sense that $E L\left(N * / p_{\alpha}-m\right) \approx 1-E L\left(N * / p_{\alpha}+m\right)$ for $m: 0<m<N * / p_{\alpha}$. The horizontal scale of Figure 2 shows values twice those of Figure 1, due to $p_{\alpha}=0.5$; that is, Figure 1 shows results in terms of "good" ASCMs, from 0 to 24 missiles fired. By comparison, Figure 2 counts "total" missiles fired, of which half will be "good" on average unless intercepted; thus the equivalent scale goes from 0 to $(24) /(0.5)=48$.

\section{Multiple Targets}

Now consider the more interesting case where there are several identical potential targets. In Figure 2 for example, the average loss curves for one target and for two targets (assuming evenly divided fire) cross over at about $N=17.2$ missiles, close but not equal to twice the equivalent deterministic breakpoint of $(9) /(0.5)=18$. For this situation I rely mostly on numerical experimentation, as the integral expressions that make up the stochastic model's equations are much harder to work with analytically than the simple algebraic equations of the deterministic model. I use an Excel spreadsheet implementation of the stochastic model from [1] to evaluate the results of using different salvo sizes. Parameters values are chosen so as to explore two dimensions: the target's defensive emphasis and the level of random variability. The levels of variability are:

- Low variability: the standard deviation of damage per hit is set at one-sixth of the mean, $\sigma_{v}=\mu_{v}(1 / 6)$, and each missile is successful with probability $p_{\alpha}=p_{z}=5 / 6$;

- Medium variability: the standard deviation of damage is two-sixths of the mean, $\sigma_{\nu}=\mu_{\nu}(2 / 6)$, and missiles hit with probability $p_{\alpha}=p_{z}=4 / 6$. These hit probabilities are similar to the empirical estimates of wartime missile accuracy in [5: $\mathrm{p} 276$ ];

- High variability: the standard deviation of damage is three-sixths of the mean, $\sigma_{v}=\mu_{v}(3 / 6)$, and missiles hit with probability $p_{\alpha}=p_{z}=3 / 6$.

The three representative categories of defensive emphasis are:

- Active defense: the enemy has strong defensive power but weak staying power. Targets have $\mathrm{E}[z]=5$ and $1 / \mu_{v}=1$; 
- Balanced defense: enemy ships have moderate defensive power and moderate staying power. Targets have $\mathrm{E}[z]=3$ and $1 / \mu_{\nu}=3$;

- Passive defense: the enemy ships have weak defensive power but strong staying power. Targets have $\mathrm{E}[z]=1$ and $1 / \mu_{\nu}=5$.

Together these two dimensions define 9 test scenarios. For example, in the high variability balanced defense scenario, the loss per non-intercepted missile has mean $\mu_{v}=$ $1 / 3$ ships and standard deviation $\sigma_{v}=(3 / 6)(1 / 3)=1 / 6$ ships. A targeted ship attempts the interception of $n_{z}=6$ incoming missiles, each with independent probability of success $p_{z}$ $=3 / 6$, so that the defensive power of a ship has an underlying binomial distribution with mean $\mathrm{E}[z]=(6)(3 / 6)=3$ missiles and a standard deviation of $(6(3 / 6)(1-3 / 6))^{1 / 2}=1.225$; as described in [1], the stochastic model approximates this binomial by a normal distribution. Offensive missiles fired at this target also have a $p_{\alpha}=3 / 6$ independent chance of success, so that a salvo of, e.g., $N=4$ ASCMs would produce an average of $(4)(3 / 6)=2$ "good" missiles with a standard deviation of $(4(3 / 6)(1-3 / 6))^{1 / 2}=1$.

Table 1 summarizes the inputs for this and the other 8 scenario combinations. Notice that the "total strength" of each target, as represented by the sum $E[z]+1 / \mu_{v}=6$, is the same in all 9 scenarios in this study.

For each of the 9 combinations of variability and defensive emphasis I use the spreadsheet to calculate a set of stochastic breakpoints and their deterministic model equivalents, and also to evaluate the effects of using these salvo sizes. Table 2 shows some of the model outputs for the scenario with high variability and balanced defenses. The first and second row, respectively, list the deterministic breakpoints $N_{1,2}$ to $N_{9,10}$ calculated using Proposition 1 and the actual stochastic breakpoints that were found via numerical search. Notice that multiplying each deterministic breakpoint value by 2 , in order to adjust for the average proportion $p_{\alpha}=3 / 6$ of offensive missiles that are "good" or well-targeted shots, provides a close estimate of the corresponding stochastic breakpoint in this particular example.

As can be seen in Table 3, the goodness of these estimates varies with the scenario being modeled. The left half of this table shows the percentage difference of the stochastic breakpoints $N_{1,2}$ from their deterministic equivalents after adjusting the latter for offensive missile accuracy $p_{\alpha}$. For example, for the high variance balanced defense scenario the relative difference between the stochastic breakpoint of 17.2 missiles and the adjusted deterministic equivalent of $(9)(6 / 3)=18$ missiles is about $(17.2-18) / 18=-4 \%$. The right half of the table shows the relative differences in values for the $N_{9,10}$ breakpoints, i.e., where it is equally beneficial to fire at 9 or 10 targets. The adjusted deterministic values tend to be good estimators of the stochastic ones to the extent that the defense is balanced, the number of targets is small, and the level of variability is low. On the other hand, the stochastic and deterministic breakpoints tend to differ to the extent that the defense is unbalanced, the number of targets is large, and the level of variability is high. For example, the $N_{9,10}$ breakpoint in the stochastic model is about $20 \%$ higher than the deterministic equivalent in the high variability active defense case. 
Figure 3 further explores this case by plotting the first 9 stochastic breakpoints $N_{1,2}$ to $N_{9,10}$ for the high variance active defense case (shown as dots) in comparison to their deterministic equivalents (shown as a straight line). A small part of the divergence in the plots occurs because the increments between breakpoints are not all equal in the stochastic model, and this causes a slight curvature. For example, $\Delta N_{9} \equiv\left(N_{9,10}-N_{8,9}\right)=$ 15.0 while $\Delta N_{2} \equiv\left(N_{2,3}-N_{1,2}\right)=15.3$. However, this departure from linearity appears to be immaterial: applying least squares regression to the 9 breakpoints yields a straight line with an extremely good fit, with $R^{2}>0.999$.

The primary difference between the plots in Figure 3 is in the steepness of their slopes. The average increment between breakpoints in the stochastic model with active defense is $\overline{\Delta N}=\left(N_{9,10}-N_{1,2}\right) / 8=15.0$ missiles, whereas the adjusted deterministic model's value is $6(6 / 3)=12.0$ for the same case. Table 4 shows these values in the upper-right and lower-right cells respectively; the values for the other scenarios appear in the remaining cells. Notice that scenarios emphasizing active defensive power all have larger average increments (and thus steeper slopes) than their deterministic equivalents, whereas the scenarios that emphasize passive staying power show smaller increments (and so shallower slopes) in the stochastic model than in the deterministic one.

One explanation for these differences in the average breakpoint increment $\overline{\Delta N}$ (as reflected in the slope of the plots) is that the stochastic model makes a greater distinction between defensive power and staying power than does the deterministic model. In mathematical terms, the stochastic model gives each of these factors a distinct distribution and includes both their means and variances in the equations, whereas the deterministic model only considers the sum of their average values. Thus the balance between these two factors matters more in the stochastic case, whereas it is mainly their total that matters in the deterministic case.

Thinking more in military terms, the breakpoint differences illustrate that missiles used to saturate defensive power serve a different purpose than missiles allocated to destroy staying power: the former is a necessary prerequisite, while the latter ("combat work" in terms of [6]) is the valued objective. Each missile that penetrates the defense can potentially cause a lot of damage to the target, depending on how "lucky" the warhead is. If many missiles hit the target, there is a possibility that the potential damage will exceed the staying power of the ship, resulting in wasteful overkill. This fact suggests that I should spread my ASCMs over as many targets as possible to maximize the expected loss. But when the targets have active defenses, there is an initial "cost" (the "price of admission") for attacking each added target, due to the need to saturate its defenses before inflicting damage. This suggests that I should focus my ASCMs on a smaller number of targets. I believe it is the varying trade-off between these two factors that results in the different average increments $\overline{\Delta N}$ for each scenario.

Figure 4 examines this idea further by plotting the average breakpoint increment $\overline{\Delta N}$ over a range of defensive power values at the high level of variability; as before, the "total strength" is kept constant at $\left(\mathrm{E}[z]+1 / \mu_{v}\right)=6$. This plot clearly shows the trend towards smaller breakpoint increments as defensive emphasis gradually switches from 
active defensive power to passive staying power. For example, the scenario with active defense $(\mathrm{E}[z]=5$ successful SAMs per target) gives $\overline{\Delta N}=15.0$ ASCMs as previously discussed. Cutting the defensive power in half to $\mathrm{E}[z]=2.5 \operatorname{good}$ SAMs while increasing staying power to compensate drops the average increment to $\overline{\Delta N}=11.4$ ASCMs.

I believe that these increments between breakpoints are of interest in part because they can be thought of as "optimal" salvo sizes per target, in the following sense. In the deterministic model the increments are all $N^{* *}$, and the average suggested salvo per target $N / t$ tends to $N^{* *}$ as $t$ gets large. That is, each additional target implies the addition of a single target's "optimal" number of missiles $N^{* *}$ to the overall salvo. In a similar manner, for any particular scenario in the stochastic model the increments average $\overline{\Delta N}$, and the average suggested salvo per target $N / t$ tends to $\overline{\Delta N}$ as $t$ gets large. Since $\overline{\Delta N}$ plays a similar role in the stochastic model as $N^{* *}$ does in the deterministic model, it seems like a sensible choice of salvo size against an individual target, if we think of that target as being one of many future potential targets.

For example, suppose that in the high variability balanced defense scenario I have the capacity available to fire up to 32 ASCMs per salvo, but there are only 2 targets currently available. The breakpoint $N_{1,2}=17.2$ indicates that I should fire at least $(17.2) /(2)=8.6 \approx 9$ ASCMs per target, but I could fire as many as $(32) /(2)=16$ per target; what value within those limits should I choose? I am arguing here that $\overline{\Delta N}=12.2$ ASCMs per target is one reasonable option. This argument assumes of course that my sole objective is to maximize average enemy losses over time, and that any enemy survivors cannot damage my ships with a salvo of their own.

Table 5 explores the impact of this rationale by showing the effect of using each $\overline{\Delta N}$ value as the salvo size against an individual target: the left side of the table shows the calculated value of $E L$, while the right side shows the probability of complete target elimination (to be discussed in the next section). In the deterministic model the equivalent salvo sizes $N^{* *}$ of course cause the complete loss of 1.00 target $100 \%$ of the time. For the stochastic model the expected losses are all lower, especially where the defense emphasizes passive staying power and/or the variability is high.

After selecting an appropriate combination of salvo size $N$ and quantity of targets $t$, it is important to spread the missile fire evenly across all the targets, approximately $N / t$ missiles each, so as to maximize the expected loss. Unlike the linear form of the deterministic model, the marginal benefit per missile in the stochastic model depends on the salvo size, as shown by the curves of Figure 2. An equal allocation of missiles tends to keep the number of missiles per target in the highest marginal return region around the adjusted $N^{*}$. As an example, consider the expected losses inflicted in the high variance balanced defense scenario (where $N_{2,3}=29.6 \approx 30$ ) when firing a total of $N=30$ missiles:

- Firing 10 missiles at each of 3 targets gives $E L=0.59+0.59+0.59=1.77$ targets;

- Firing 15 at each of 2 targets similarly gives $E L=0.87+0.87+0.00=1.74$ targets;

- Firing 20 at one and 10 at another gives only $E L=0.97+0.59+0.00=1.56$ targets. 


\section{Standard Deviation Of Losses}

I now wish to briefly discuss the standard deviation of inflicted losses $S L$, as this can be interpreted as a measure of the "risky-ness" of results. Figure 5 illustrates the approximately bell-shaped curves of $S L$ for a range of salvo sizes evenly spread across 1 , 2 , or 3 targets in the high variance balanced defense case. Note that the region in which $S L$ is highest also tends to be the region that yields the highest marginal gains in $E L$ from increased salvo size. For example, when firing at a single target, the peak of the $S L$ curve (in Figure 5) and the inflection point of the EL curve (in Figure 2) are both close to $N=9$ missiles; a similar pattern can be seen in the corresponding pairs of curves for the 2- and 3 -target cases. Thus the salvo sizes that provide increased benefits or "returns" in terms of $E L$ also tend to come with increased uncertainty or "risk" in terms of $S L$.

In a similar vein, the bottom two rows of Table 2 display the calculated values of $S L$ when choosing between $t$ and $t+1$ targets at the breakpoints $N_{t, t+1}$. For example, while a salvo of $N_{2,3}=29.6 \approx 30$ missiles will give about the same average loss $E L$ when fired at either 2 or 3 targets, the standard deviation when fired at 2 targets is only $S L=0.13$ ships, while that for 3 targets is almost twice as large at $S L=0.23$. As the table shows, this difference is relatively large for small values of $t$ but declines as $t$ increases. Thus it may be worthwhile sometimes to fire at slightly fewer targets than what the breakpoints would suggest, and thereby accept a slightly lower expected loss $E L$, in order to have a much lower standard deviation $S L$ and so more certainty about the results.

\section{The Stochastic Model: Probability Of Elimination}

\section{One Target}

This section considers an alternative measure of attack effectiveness: the probability of elimination $P_{\text {elim }}$ of an enemy target. In the deterministic model, a salvo of $N^{* *}$ missiles achieves a complete firepower kill on a single target $100 \%$ of the time, whereas in the stochastic model the equivalent quantity of missiles (after adjusting for the probability of hit, so $N^{* *} / p_{\alpha}$ ) provides roughly a $P_{\text {elim }}=50 \%$ chance of complete success. About half of the time a salvo of that size causes more than enough damage to eliminate it (overkill), but the other half of the time the damage is only partial.

My approach in this section borrows another idea from stochastic inventory modeling. Faced with random customer demand, a business may hold enough product to satisfy the average demand per period, plus an additional quantity called safety stock sufficient to achieve management's chosen cycle service level: the probability of meeting all demand that occurs each period (as in [4: Ch 11]).

In this spirit, assume that the attacker will specify a desired probability of enemy elimination $q$ as their performance goal. To ensure that $P_{\text {elim }} \geq q$ they can calculate a safety margin of extra missiles $M$ to fire at the target, over and above the basic quantity required to get $P_{\text {elim }}=50 \%$. For the general case where offensive, defensive and staying 
power all exhibit random variation, the size of the safety margin can be found via numerical search. In those special cases where only one of these components has significant variability, it is possible to derive the safety margin analytically. The following proposition describes these special cases; the symbol $\Phi(\cdot)$ therein represents the standard normal cumulative distribution function and $\Phi^{-1}(\cdot)$ its inverse.

Proposition 3. To eliminate an enemy target with probability $q \geq 50 \%$, an attacker needs to fire a safety margin of $M$ extra missiles as follows.

(a) If the only significant random variability is due to the loss suffered per missile:

$$
M_{v}=\frac{\Phi^{-1}(q)}{p_{\alpha}} \Phi^{-1}(q) \frac{\sigma_{v}{ }^{2}}{\mu_{v}^{2}}\left(\left\{B \frac{\mu_{v}}{\left\{\Phi^{-1}(q)\right\}^{2} \sigma_{v}{ }^{2}}+\frac{1}{4}\right\}^{1 / 2}+\frac{1}{2}\right) .
$$

(b) If the only significant random variability is due to offensive power:

$$
M_{\alpha}=\frac{\Phi^{-1}(q)}{p_{\alpha}} \Phi^{-1}(q)\left(1-p_{\alpha}\right)\left(\left\{B \frac{\left(n_{z} p_{z}+1 / \mu_{v}\right)}{\left\{\Phi^{-1}(q)\right\}^{2}\left(1-p_{\alpha}\right)}+\frac{1}{4}\right\}^{1 / 2}+\frac{1}{2}\right)
$$

(c) If the only significant random variability is due to defensive power:

$$
M_{z}=\frac{\Phi^{-1}(q)}{p_{\alpha}}\left\{B n_{z} p_{z}\left(1-p_{z}\right)\right\}^{1 / 2}
$$

The sizes of these safety margins are parameter dependent; in my numerical trials the margin due to offensive power tended to be the largest in magnitude while that due to damage per missile tended to be the smallest, so that typically $\left|M_{\alpha}\right|>\left|M_{z}\right|>\left|M_{v}\right|$. Table 5 shows some safety margins for the balanced defense high variance scenario, for which $P_{\text {elim }}=50 \%$ is achieved at $N=11$ missiles. These were calculated numerically using an Excel spreadsheet implementation of the stochastic salvo equations. For example, the safety margin for an attack with a $q=90 \%$ chance of enemy elimination (so that $\Phi^{-1}(q)=$ 1.28 standard deviations) is $M_{v}=1.5$ missiles if the only significant variation is due to the damage per hit. This means that a total of $N=11+1.5=12.5$ missiles is required. By comparison, when all model components have random variation the needed safety margin is $M=7.3$ missiles and the salvo size is $N=11+7.3=18.3 \approx 19$.

It can be informative to break down this salvo of 19 missiles into its components:

- $1 / \mu_{\nu}=3$ average ASCM hits cause enough damage to knock out the targeted ship;

- $\mathrm{E}[z]=3$ added average ASCMs are needed to saturate the target's average defensive power so that other ASCMs can penetrate; 
- 6 more ASCMs provide an allowance for their average reliability of $p_{\alpha}=1 / 2$;

- 7 extra ASCMs are needed to increase the probability of success to at least $q=90 \%$ given the inherent variability of missile combat.

Thus in this example while just 3 missiles are theoretically sufficient to destroy the target (e.g., on a peacetime firing range), Red may need to fire more than six times that number in wartime to be confident of success.

The columns of Table 6 show how the safety margins quickly grow as $q$ approaches $100 \%$, as each increase in the specified probability of success beyond $50 \%$ requires increasingly more firepower. For example, boosting the safety margin $M$ from 0 to 2.2 missiles raises the probability of success from $50 \%$ to $67 \%$, a 17 -point improvement. By contrast a further addition of 2.3 missiles, from 2.2 to 4.5 , yields only a 13-point improvement, from $67 \%$ to $80 \%$.

This leads to the question of what probability $q$ to choose and thus what size of safety margin to employ; that is, how much certainty "should" an attacker want? Clearly this will depend in part on the attacker's mission. A flotilla that needs to quickly clear a sea lane in advance of an oncoming convoy might prefer a relatively high probability $q$, whereas a small force on a raiding mission might accept a smaller $q$ and fire fewer missiles per salvo so as to preserve its ammunition for other targets.

Another possible answer comes from the incremental salvo sizes $\overline{\Delta N}$ that were calculated earlier. For example, in the high variance active defense scenario, the calculated value of $\overline{\Delta N}=15.0$ missiles (from Table 4) yields a probability of elimination of $P_{\text {elim }}=78 \%$ when fired at a single target, as shown in the right half of Table 5 . This suggests that if the only goal is to maximize average enemy losses over the course of many attacks, then one might specify $q=78 \%$ for each individual attack in that set. Note that the scenarios with passive defenses all imply the use of negative safety margins; e.g., in the high variance passive defense case a salvo of $N=\overline{\Delta N}=8.9$ missiles indicates a margin of $M=8.9-11=-2.1$ missiles and thus $q=29 \%$. This may seem rather low, but note that the average enemy loss is still fairly high, at about 0.65 targets; so survivors would be common but heavily damaged. In inventory analysis an equivalent negative safety stock situation arises when a business deliberately keeps less stock on hand than needed to meet average demand, and thereby reduces its risk of having unsold leftovers. Of course it is unlikely that a navy would have the long-run average rate of enemy elimination as its only goal.

\section{Multiple Targets}

The last comment on this topic relates to the need for larger safety margins for multiple targets. If each of $t$ targets has an independent probability $q$ of elimination, than the probability of all of them being eliminated simultaneously is only $q^{t}<q$. For example, to be $90 \%$ assured of eliminating 3 targets at once, an attacker needs a $(0.90)^{1 / 3}$ $=96.5 \%$ chance of eliminating each one; thus for the high variance balanced defense case the safety margin for each target would climb from 7.3 to 10 missiles. 


\section{Discussion}

Underlying many of the results in this paper is a trade-off between choosing too many targets and choosing too few. If the attacker engages fewer targets, they reduce the "wasting" of missiles to saturate defenses, and their larger salvo size per target provides reduced variability of losses, greater safety margins, and more certainty of results. On the other hand, engaging more targets reduces the "wasting" of missiles on target overkill, and can cause more damage on average, but accepts more "risk" or uncertainty in the outcome. This trade-off is particularly important when there are only a few targets engaged at once; the differences gradually decline as more targets are added.

One interesting result in this study is how the nature of the target's defense, active versus passive, significantly influences this tactical trade-off in the presence of variability. The stochastic model shows that the breakpoints for targets with predominantly passive staying power are smaller, implying that the attacker should spread their fire over more targets: most of the missiles will contribute towards enemy damage, and by firing at more targets the attacker will inflict more total damage on average. For targets with predominantly active defensive power the breakpoints are higher, suggesting the attacker should focus on fewer targets; this reduces "under-kill" and helps assure good results against the targets chosen.

Throughout this work I phrase my analysis in terms of the number of missiles $N$ to launch at the moment of attack, but it is easy to convert these figures to a corresponding number of warships. For example, if each Red ship carries 4 ASCMs, then Red could deliver a desired salvo of $N=20$ missiles by deploying 20/4 $=5$ such vessels into battle. This alternative frame of reference may be more appropriate for a theatre commander deciding how many ships to send into battle.

\section{Conclusions \& Limitations}

If a naval commander's primary aim is to "attack effectively first" [5: p xxv] then it is important to be able to analyze the effectiveness of planned attacks. In this paper I studied attack effectiveness in the context of the salvo combat model. I began with the deterministic version of the model and analytically derived an optimality relation between the number of targets to attack and the number of missiles to fire. For the more complex stochastic model, I used a numerical study to explore two complementary performance measures, the average enemy loss and the probability of enemy elimination.

When the military objective was to maximize average enemy losses, it was not surprising to find that a simple linear structure exists for optimal firing in the deterministic model, given the simplicity of its salvo equations. It was, however, a pleasant surprise to find that the same linear structure also appears to hold (with different parameters) as a good approximation for the more complex stochastic model. I argued 
that it was possible to interpret the incremental number of missiles fired (the "slope" of this linear relation) as being in some respects an "optimal" salvo size per target.

When considering the probability of enemy elimination, I showed that the concept of having a safety margin of extra missiles to fire is relatively straightforward to adapt from inventory theory. The numerical work demonstrated that the appropriate salvo sizes under either performance measure depend on the relative balance between the target's active and passive strength. Active defensive power requires larger salvos focused on fewer targets, while passive staying power encourages smaller salvos against more targets, even when the total or combined defensive strength is the same in both cases.

Although I believe these results will be of interest to both researchers and naval analysts, I must note that there are many limitations to the methodology used. The most important caveat is that all of the work herein is based upon the salvo models of [1] and [6], and so makes the same assumptions as those models. Thus the results are meaningful to real naval officers only to the extent that those simple models capture the essential aspects of real naval combat. For example, the models assume that all enemy ships are identical and that the attacker has full knowledge of their defenses, whereas in practice each fleet may employ a mixture of ship types and do its best to limit the opponent's intelligence gathering. The defender is likewise assumed to have perfect knowledge when allocating their defensive power, and can thereby maximize the number of missiles they intercept. Another implicit assumption is that the attacker can choose exactly which target(s) to fire upon and hopefully hit, whereas wartime conditions might result in more randomized targeting due to, e.g., limited scouting information as in [8], or the imperfect selectivity of missile guidance systems as in [7].

\section{Acknowledgements}

I would like to thank three anonymous referees for their suggestions on improving this paper. During this research the author was funded in part by a Discovery Grant from Canada’s Natural Sciences \& Engineering Research Council (NSERC).

\section{References}

[1] Armstrong MJ, 2005, "A stochastic salvo model for naval surface combat", Operations Research 53, \#5, 830-841.

[2] Armstrong MJ, 2004, "Effects of lethality in naval combat models", Naval Research Logistics 51, 28-43.

[3] Armstrong MJ \& Powell MB, 2005, "A salvo combat analysis of the Battle of the Coral Sea", Military Operations Research 10, \#4, 27-38.

[4] Chopra S, Meindl P, 2004, Supply Chain Management, $2^{\text {nd }}$ Ed, Pearson Education Inc, Upper Saddle River, NJ, USA. 
[5] Hughes WP, 2000, Fleet Tactics and Coastal Combat, $2^{\text {nd }}$ Ed, Naval Institute Press, Annapolis, MA, USA.

[6] Hughes WP, 1995, “A salvo model of warships in missile combat used to evaluate their staying power”, Naval Research Logistics 42, 267-289.

[7] Hura M, Miller D, 1985, "Cruise missile warfare", United States Naval Institute Proceedings, Oct, 97-101.

[8] Lucas TW, McGunnigle JE, 2003, “When is model complexity too much?”, Naval Research Logistics 50, 197-217.

[9] Wagner DH, Mylander WC, Sanders TJ, 1999, Naval Operations Analysis, $3^{\text {rd }}$ Ed, Naval Institute Press, Annapolis, MA, USA.

\section{Appendix}

\section{Derivations For Proposition 1: Deterministic Model}

Part (a) is a statement of the obvious but not always attainable ideal.

For part (b) first consider whether to shoot at 1 target. To saturate the target's defense Red needs to fire $N_{0,1}=z$ missiles, in which case the loss is zero whether Red fires at that target or not. As soon as the salvo size exceeds $N_{0,1}$, Red should shoot at the target because they would begin to damage it.

Next let Red decide whether to engage $t$ or $t+1$ targets. A salvo of $N=(t)(z+1 / v)$ missiles exactly eliminates $t$ targets; to inflict greater losses would require engaging an added target. At a salvo size of $N_{t, t+1}=\{z+t(z+1 / v)\}$ Red is indifferent between $t$ or $t+1$ targets, as some missiles are wasted either way; but as soon as $N$ exceeds $N_{t, t+1}$ they would be better to shoot at $t+1$ targets.

Part (c) is derived by re-arranging the relation in part (b) to solve for $t$.

For part (d), divide $N_{t-1, t}$ from part (b) by $t$ and proceed as follows.

$$
N_{t-1, t} / t=\{z+(t-1)(z+1 / v)\} / t=z+(1 / v)(t-1) / t \geq z+(1 / v) / 2=N^{*}
$$

The other results are obtained by taking the limit as $t \rightarrow \infty$.

$$
\lim _{t \rightarrow \infty}\left(\frac{(t-1)(z+1 / v)+z}{t}\right)=z+1 / v-\lim _{t \rightarrow \infty}\left(\frac{1 / v}{t}\right)=N * *
$$

\section{Notation For The Stochastic Model}


Let $G(l), g(l), \mu$ and $\sigma^{2}$ represent the cumulative distribution function, probability density function, mean and variance, respectively, of the nominal loss by Blue, where

$$
g(l)=\frac{1}{\sqrt{2 \pi \sigma^{2}}} e^{\frac{-(l-\mu)^{2}}{2 \sigma^{2}}}, \quad \frac{\partial}{\partial \mu} g(l)=\frac{l-\mu}{\sigma^{2}} g(l), \quad \frac{\partial}{\partial \sigma} g(l)=\frac{(l-\mu)^{2}-\sigma^{2}}{\sigma^{3}} g(l) .
$$

The nominal loss follows a normal distribution (not truncated) and so could be negative or very large. The actual loss is restricted to the range 0 to $B$ by assigning any probability mass below 0 to 0 , and likewise by assigning any probability mass above $B$ to $B$. This leads to the following expressions from [1].

$$
\begin{gathered}
E L=\int_{0}^{B} l g(l) d l+\int_{B}^{\infty} B g(l) d l=\int_{0}^{B}(1-G(l)) d l=\int_{0}^{B} \int_{l}^{\infty} g(t) d t d l \\
\mu=E\left[N e t_{A B}\right] E[v]=\left(N p_{\alpha}-B n_{z} p_{z}\right) \mu_{v} \\
\sigma^{2}=\left(N p_{\alpha}-B n_{z} p_{z}\right) \sigma_{v}^{2}+\left(N p_{\alpha}\left(1-p_{\alpha}\right)+B(1-p) n_{z} p_{z}\right) \mu_{v}{ }^{2} \\
-2 \sigma_{v}{ }^{2}\left(N p_{\alpha}-B n_{z} p_{z}\right) G_{N e t A B}(0)+2 \sigma_{v}{ }^{2}\left(N p_{\alpha}\left(1-p_{\alpha}\right)+B(1-p) n_{z} p_{z}{ }^{2}\right) g_{N e t A B}(0)
\end{gathered}
$$

The following derivations leave out the continuity correction from the stochastic salvo equations of [1] so as to make the expressions easier to interpret.

\section{Derivations For Proposition 2: Expected Loss}

For part (a) take the first and second derivative of $E L$ with respect to $\mu$.

$$
\begin{aligned}
& \frac{\partial}{\partial \mu} E L=\frac{1}{\sigma^{2}} \int_{0}^{B} \int_{l}^{\infty}(t-\mu) g(t) d t g(l) d l \\
& \quad=\frac{1}{\sigma^{2}} \int_{0}^{B}\left\{\mu(G(\infty)-G(l))-\sigma^{2}(g(\infty)-g(l))-\mu(G(\infty)-G(l))\right\} d l=\int_{0}^{B} g(l) d l>0 \\
& \frac{\partial^{2}}{\partial \mu^{2}} E L=\frac{1}{\sigma^{2}} \int_{0}^{B}(l-\mu) g(l) d l=g(0)-g(B)
\end{aligned}
$$

Because $g(l)$ is a symmetric distribution the last expression is zero when $\mu=B / 2(50 \%$ loss), indicating an inflection point. For $\mu<B / 2$ it is positive and $E L$ is convex increasing, while for $\mu>B / 2$ it is negative and $E L$ is concave increasing.

For part (b) take the derivative of $E L$ with respect to $\sigma$. 


$$
\begin{aligned}
& \frac{\partial}{\partial \sigma} E L=\frac{1}{\sigma^{3}} \int_{0}^{B} \int_{l}^{\infty}\left(t^{2}-2 t \mu+\mu^{2}-\sigma^{2}\right) g(t) d t d l \\
& =\frac{1}{\sigma^{3}} \int_{0}^{B}\left\{\left(\mu^{2}+\sigma^{2}\right)(G(\infty)-G(l))-\sigma^{2}(\langle\infty+\mu\rangle g(\infty)-\langle l+\mu\rangle g(l))\right\} d l \\
& +\frac{1}{\sigma^{3}} \int_{0}^{B}\left(\mu^{2}(G(\infty)-G(l))-2 \mu\left\{\mu(G(\infty)-G(l))-\sigma^{2}(g(\infty)-g(l))\right\}-\sigma^{2}(G(\infty)-G(l))\right) d l \\
& =\frac{1}{\sigma} \int_{0}^{B}(l-\mu) g(l) d l=\frac{1}{\sigma}\left\{\mu(G(B)-G(0))-\sigma^{2}(g(B)-g(0))-\mu(G(B)-G(0))\right\} \\
& =\sigma\{g(0)-g(B)\}
\end{aligned}
$$

Because $g(l)$ is symmetric, the derivative is zero when $\mu=B / 2$ (50\% loss), indicating an extreme point. For $\mu<B / 2$ it is positive and $E L$ is increasing, while for $\mu>B / 2$ it is negative and $E L$ is decreasing.

For part (c) combine the results of parts (a) and (b) along with the fact that offensive power's mean $N p_{\alpha}$ and variance $N p_{\alpha}\left(1-p_{\alpha}\right)$ are both linearly increasing in $N$.

\section{Derivations For Proposition 3: Safety Margins}

To get $P_{\text {elim }} \geq q$, start by using the normality relation

$$
q=\Phi(\{\mu-B\} / \sigma) \text { so that } \Phi^{-1}(q)=\{\mu-B\} / \sigma .
$$

Square both sides and rearrange to get

$$
\left\{\Phi^{-1}(q)\right\}^{2} \sigma^{2}-\{\mu-B\}^{2}=0 .
$$

For part (a) where damage per missile has mean $\mu_{v}$ and variance $\sigma_{v}{ }^{2}$, simplified $\mu$ and $\sigma$ are obtained as follows.

$$
\mu=E\left[\sum^{N e t A B} v\right]=\left(N p_{\alpha}-B n_{z} p_{z}\right) \mu_{v} \quad \sigma^{2}=\operatorname{Var}\left[\sum^{N e t A B} v\right]=\left(N p_{\alpha}-B n_{z} p_{z}\right) \sigma_{v}{ }^{2}
$$

Substitute these into Equation A1.

$$
\begin{gathered}
\left\{\Phi^{-1}(q)\right\}^{2}\left(N p_{\alpha}-B n_{z} p_{z}\right) \sigma_{v}{ }^{2} \\
-\left\{N^{2} p_{\alpha}^{2} \mu_{v}{ }^{2}+B^{2} n_{z}{ }^{2} p_{z}^{2} \mu_{v}{ }^{2}+B^{2}-2 B N p_{\alpha} \mu_{v}+2 B^{2} n_{z} p_{z} \mu_{v}-2 N p_{\alpha} B n_{z} p_{z} \mu_{v}^{2}\right\}=0
\end{gathered}
$$


Re-arrange into quadratic form and then solve for $N$.

$$
\begin{gathered}
N^{2}\left\{-p_{\alpha}{ }^{2} \mu_{v}{ }^{2}\right\}+N\left\{\Phi^{-1}(q)^{2} p_{\alpha} \sigma_{v}{ }^{2}+2 B p_{\alpha} \mu_{v}\left(1+n_{z} p_{z} \mu_{v}\right)\right\} \\
+\left\{-\Phi^{-1}(q)^{2} B n_{z} p_{z} \sigma_{v}{ }^{2}-B^{2}-B^{2} n_{z}{ }^{2} p_{z}{ }^{2} \mu_{v}{ }^{2}-2 B^{2} n_{z} p_{z} \mu_{v}\right\}=0 \\
N=\frac{2 B p_{\alpha} \mu_{v}\left(1+\mu_{v} n_{z} p_{z}\right)}{2 p_{\alpha}{ }^{2} \mu_{v}{ }^{2}}+\frac{\left(\Phi^{-1}(q)\right)^{2} p_{\alpha} \sigma_{v}{ }^{2}}{2 p_{\alpha}{ }^{2} \mu_{v}{ }^{2}} \\
+\frac{\left(\left\{\left(\Phi^{-1}(q)\right)^{2} p_{\alpha} \sigma_{v}{ }^{2}-2 B p_{\alpha} \mu_{v}\left(1+\mu_{v} n_{z} p_{z}\right)\right\}^{2}-4 p_{\alpha}{ }^{2} \mu_{v}{ }^{2}\left\{\left(\Phi^{-1}(q)\right)^{2} B n_{z} p_{z} \sigma_{v}{ }^{2}+B^{2}\left(1+\mu_{v} n_{z} p_{z}\right)^{2}\right\}\right)^{1 / 2}}{2 p_{\alpha}{ }^{2} \mu_{v}{ }^{2}}
\end{gathered}
$$

The first term gives $P_{\text {elim }}=50 \%$; rearrange the remaining terms to form $M_{v}$.

For part (b) offensive power has mean $N p_{\alpha}$ and variance $N p_{\alpha}\left(1-p_{\alpha}\right)$, so $\mu$ and $\sigma$ are

$$
\mu=E\left[\sum^{N e t A B} v\right]=\left(N p_{\alpha}-B n_{z} p_{z}\right) \mu \quad, \quad \sigma^{2}=\operatorname{Var}\left[\sum^{N e t A B} v\right]=N p_{\alpha}\left(1-p_{\alpha}\right) v^{2}
$$

Substitute these into Equation A1, then re-arrange and solve for $N$ as in part (a) above.

$$
\begin{aligned}
\left\{\Phi^{-1}(q)\right\}^{2} N p_{\alpha}\left(1-p_{\alpha}\right) v^{2}-\left\{N^{2} p_{\alpha}{ }^{2} v^{2}+B^{2} n_{z}^{2} p_{z}^{2} v^{2}+B^{2}-2 N p_{\alpha} v^{2} B z-2 N p_{\alpha} v B+2 B^{2} n_{z} p_{z} v\right\}=0 \\
N=\frac{\left(\left\{\Phi^{-1}(q)\right\}^{2}\left(1-p_{\alpha}\right) v+2 B v n_{z} p_{z}+2 B\right)}{2 p_{\alpha} v} \\
+\frac{\left\{\left(\left\{\Phi^{-1}(q)\right\}^{2}\left(1-p_{\alpha}\right) v+2 B v n_{z} p_{z}+2 B\right)^{2}+4\left(-B^{2} v^{2} n_{z}{ }^{2} p_{z}{ }^{2}-B^{2}-2 B^{2} v n_{z} p_{z}\right)\right\}^{1 / 2}}{2 p_{\alpha} v}
\end{aligned}
$$

For part (c) defensive power per ship has mean $n_{z} p_{z}$ and variance $n_{z} p_{z}\left(1-p_{z}\right)$ so $\mu$ and $\sigma$ are as follows.

$$
\mu=E\left[\sum^{N e t A B} v\right]=\left(N p_{\alpha}-B n_{z} p_{z}\right) v \quad \sigma^{2}=\operatorname{Var}\left[\sum^{N e t A B} v\right]=B n_{z} p_{z}\left(1-p_{z}\right) v^{2}
$$

Substitute these into Equation A1 and re-arrange to solve for $N$ as in part (a).

$$
\left\{\Phi^{-1}(q)\right\}^{2} B v^{2} n_{z} p_{z}\left(1-p_{z}\right)-\left(N^{2} p_{\alpha}^{2} v^{2}+B^{2} n_{z}^{2} p_{z}^{2} v^{2}+B^{2}-2 N p_{\alpha} v^{2} B n_{z} p_{z}-2 N p_{\alpha} v B+2 B^{2} n_{z} p_{z} v\right)=0
$$




$$
\begin{aligned}
& N=\frac{\left(B v n_{z} p_{z}+B\right)}{p_{\alpha} v} \\
& +\frac{\left\{\left(B^{2} v^{2} n_{z}{ }^{2} p_{z}{ }^{2}+B^{2}+2 B^{2} v n_{z} p_{z}\right)+\left(B M^{2} v^{2} n_{z} p_{z}\left(1-p_{z}\right)-B^{2} v^{2} n_{z}{ }^{2} p_{z}{ }^{2}-B^{2}-2 B^{2} v n_{z} p_{z}\right)\right\}^{1 / 2}}{p_{\alpha} v}
\end{aligned}
$$

\section{Further Numerical Work For The Stochastic Model}

A second set of calculations was conducted with all of the target parameters being twice as large; i.e., with $\mathrm{E}[z]+1 / \mu_{v}=12$ rather than 6 . While the details varied, the overall trends were the same as in the results reported herein. 
Figure 1. Losses inflicted when spreading fire equally across 1 (solid line), 2 (dashes), or 3 (dots) targets in the deterministic model with balanced defenses.

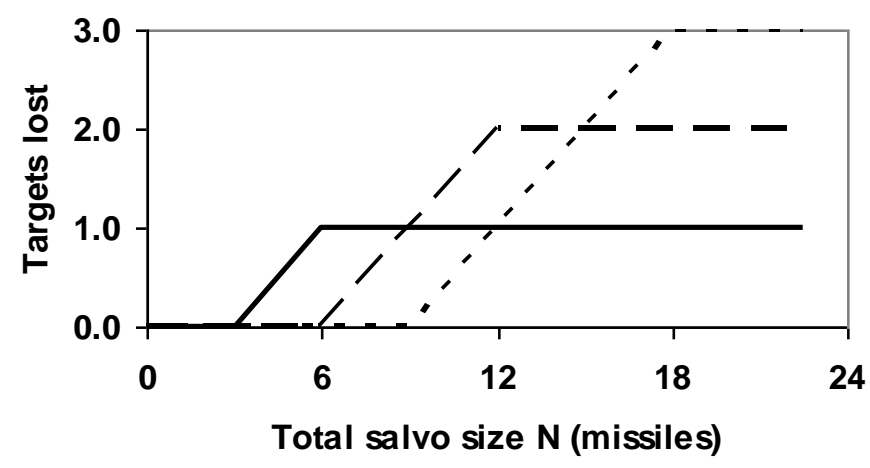

Figure 2. Expected losses $E L$ when spreading fire equally across 1,2 or 3 targets in the stochastic model for the balanced defense high variability case.

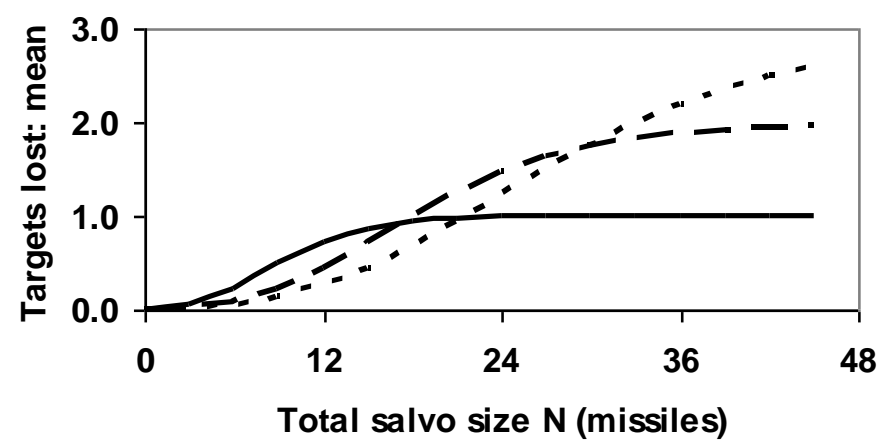

Figure 3. Comparison of the adjusted deterministic breakpoints (straight line) to the stochastic breakpoints for the active defense high variability case (square dots).

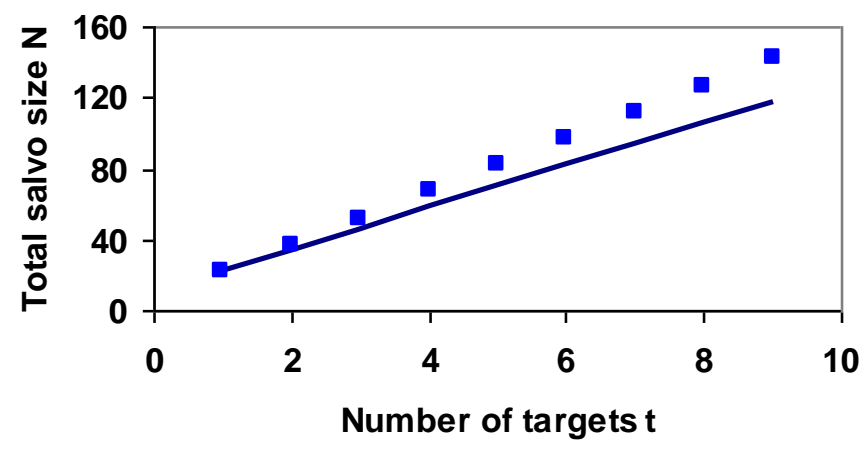


Figure 4. Average breakpoint increments $\overline{\Delta N}$ as the defensive emphasis is varied in the stochastic model with high variability.

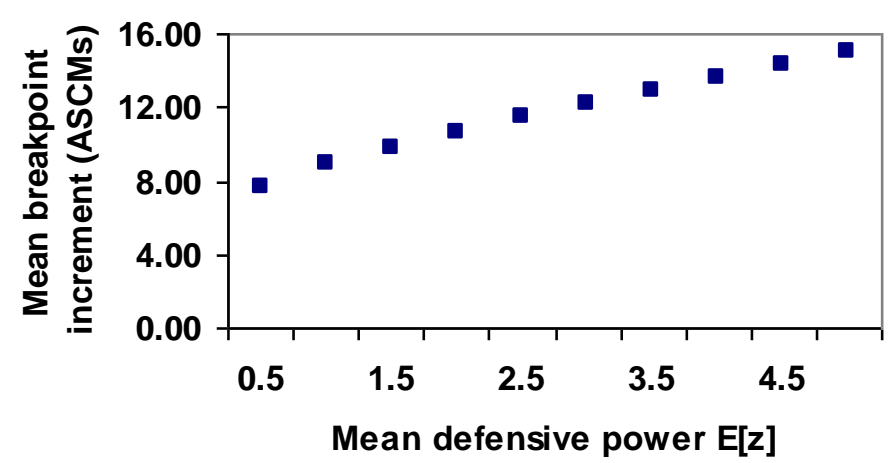

Figure 5. Standard deviation of losses $S L$ when spreading fire equally across 1,2 or 3 targets in the stochastic model for the balanced defense high variability case.

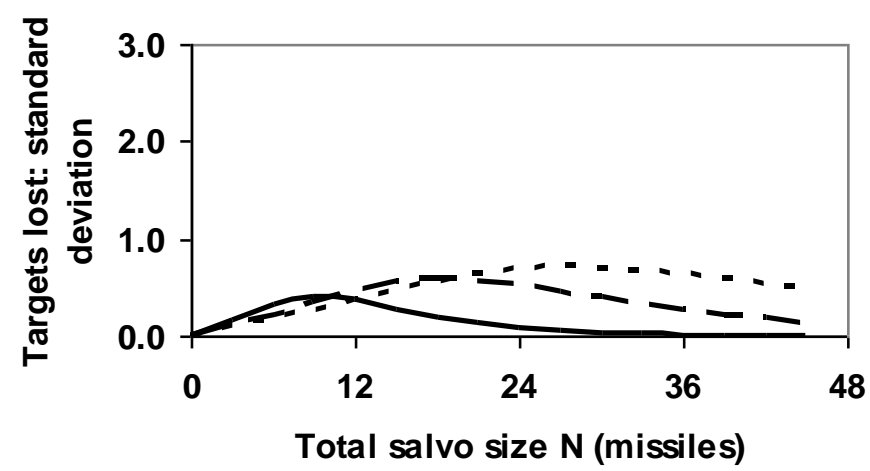

Table 1. Stochastic model input data for the 9 scenarios of the numerical study for offensive missile accuracy $p_{\alpha}$, defensive power per ship $z$, and damage per missile $v$.

\begin{tabular}{|lccccccccc|}
\hline Variability & & Low & \multicolumn{3}{c|}{ Medium } & \multicolumn{3}{c|}{ High } \\
\hline Defense & Active & Balanced & Passive & Active & Balanced & Passive & Active & Balanced & Passive \\
\hline$p_{\alpha}$ & 0.833 & 0.833 & 0.833 & 0.667 & 0.667 & 0.667 & 0.500 & 0.500 & 0.500 \\
E $[z]$ & 5 & 3 & 1 & 5 & 3 & 1 & 5 & 3 & 1 \\
Std dev $[z]$ & 0.913 & 0.707 & 0.408 & 1.291 & 1.000 & 0.577 & 1.581 & 1.225 & 0.707 \\
$\mu_{v}$ & 1 & 3 & 5 & 1 & 3 & 5 & 1 & 3 & 5 \\
$\sigma_{v}$ & 0.167 & 0.500 & 0.833 & 0.333 & 1.000 & 1.667 & 0.500 & 1.500 & 2.500 \\
\hline
\end{tabular}


Table 2. Results for the high variability balanced defense case: breakpoints $N_{\mathrm{t}, \mathrm{t}+1}$, expected loss $E L$, and standard deviations of loss $S L$ for $t$ targets and $t+1$ targets.

\begin{tabular}{|lccccccccc|}
\hline$t, t+1$ & 1,2 & 2,3 & 3,4 & 4,5 & 5,6 & 6,7 & 7,8 & 8,9 & 9,10 \\
\hline$N_{t, t+1}$ Deterministic & 9 & 15 & 21 & 27 & 33 & 39 & 45 & 51 & 57 \\
\hline$N_{t, t+1}$ Stochastic & 17.2 & 29.6 & 41.8 & 54.0 & 66.1 & 78.2 & 90.3 & 102.4 & 114.4 \\
$E L$ & 0.46 & 0.57 & 0.62 & 0.65 & 0.66 & 0.67 & 0.68 & 0.69 & 0.69 \\
$S L$ for $t$ & 0.28 & 0.23 & 0.20 & 0.17 & 0.16 & 0.14 & 0.13 & 0.13 & 0.12 \\
$S L$ for $t+1$ & 0.11 & 0.13 & 0.13 & 0.13 & 0.12 & 0.12 & 0.11 & 0.11 & 0.10 \\
\hline
\end{tabular}

Table 3. Relative \% difference in the breakpoints $N_{1,2}$ and $N_{9,10}$ of the stochastic model as compared to their adjusted equivalents from the deterministic model.

\begin{tabular}{|lcccccc|}
\hline & \multicolumn{3}{c}{$N_{1,2}$} & & \multicolumn{3}{c|}{$N_{9,10}$} \\
\hline & Low & Medium & High & Low & Medium & High \\
\hline Active & $0 \%$ & $-1 \%$ & $-2 \%$ & $+17 \%$ & $+21 \%$ & $+20 \%$ \\
Balanced & $0 \%$ & $-2 \%$ & $-4 \%$ & $+3 \%$ & $+3 \%$ & $+0 \%$ \\
Passive & $-2 \%$ & $-8 \%$ & $-14 \%$ & $-12 \%$ & $-19 \%$ & $-25 \%$ \\
\hline
\end{tabular}

Table 4. Average increments (number of missiles between breakpoints) $\overline{\Delta N}$ for the stochastic model and their adjusted equivalents from the deterministic model.

\begin{tabular}{|lccr|}
\hline & Low & Medium & High \\
\hline Active & 8.7 & 11.3 & 15.0 \\
Balanced & 7.4 & 9.3 & 12.2 \\
Passive & 6.2 & 7.2 & 8.9 \\
\hline Adjusted Deterministic & 7.2 & 9.0 & 12.0 \\
\hline
\end{tabular}

Table 5. Expected loss and probability of elimination in the stochastic model when using the corresponding average breakpoint increment $\overline{\Delta N}$ as the salvo size against one target.

\begin{tabular}{|lcccccc|}
\hline & \multicolumn{3}{c}{ Expected Loss } & \multicolumn{3}{c|}{$\begin{array}{c}\text { Probability of } \\
\text { Elimination }\end{array}$} \\
\hline & Low & Medium & High & Low & Medium & High \\
\hline Active & 0.89 & 0.83 & 0.78 & $89 \%$ & $83 \%$ & $78 \%$ \\
Balanced & 0.87 & 0.80 & 0.74 & $70 \%$ & $65 \%$ & $60 \%$ \\
Passive & 0.82 & 0.73 & 0.65 & $38 \%$ & $32 \%$ & $29 \%$ \\
\hline
\end{tabular}

Table 6. Safety margins needed to provide at least probability $q$ of complete elimination for the high variability balanced defense scenario.

\begin{tabular}{|lcccccc|}
\hline Probability $q$ & $33 \%$ & $50 \%$ & $67 \%$ & $80 \%$ & $90 \%$ & $95 \%$ \\
\hline$M_{v}$ & -0.2 & 0.0 & 0.4 & 0.8 & 1.5 & 2.1 \\
$M_{z}$ & -1.3 & 0.0 & 1.4 & 2.7 & 4.1 & 5.2 \\
$M_{\alpha}$ & -1.3 & 0.0 & 1.6 & 3.2 & 5.2 & 7.0 \\
$M$ & -1.9 & 0.0 & 2.2 & 4.5 & 7.3 & 9.9 \\
\hline
\end{tabular}

